\title{
Statistical processing of West Africa wave directional spectra time-series into a climatology of swell events
}

\author{
Michel Olagnon ${ }^{\mathrm{a}, *}, \mathrm{~K}$. Agbéko Kpogo-Nuwoklo ${ }^{\mathrm{a}, \mathrm{b}}$, Zakoua Guédé ${ }^{\mathrm{a}}$ \\ ${ }^{a}$ Ifremer Centre de Brest, B.P. 70, F-29280 Plouzané, France \\ ${ }^{\mathrm{b}}$ Université d'Abomey-Calavi, Cotonou, Bénin \\ *: Corresponding author : Michel Olagnon, tel.: + 33298224144 ; email address : molagnon@ifremer.fr ; \\ kkpogonu@ifremer.fr ; zguede@ifremer.fr
}

\begin{abstract}
:
Accurate estimation of long-term sea conditions is a major issue for the design of coastal and offshore structures, for the preparation of marine operations, and for other applications such as marine energy, and coastal erosion. It requires, on the one hand, proper parametric models of the sea state spectra and the statistics of the parameters, and on the other hand, representations of their time evolutions. In some locations such as West Africa, sea conditions are complex with wave spectra showing many well separated peaks corresponding to several swells and wind sea. The present study focuses on swell at a West Africa location. First, a time-consistent triangular model is assessed for the spectral shapes of the swell components. Then statistical analysis of the time-histories of those components is carried out in connection with the storms at their source. A model that is triangular for $H_{s}$ and a linear trend for period and direction is found appropriate for the time-histories of those parameters within a storm event. Using the empirical distributions of the characteristics of the individual events parameters, it is shown that arbitrary long durations of the swell climate may be reconstructed preserving the main observed statistical properties..
\end{abstract}

\section{Highlights}

- We model individual wave directional spectra as the combination of several systems. A parametric shape suited to swell is proposed for swell systems - The time-series of systems is modeled as swell events corresponding to remote storms Reconstruction of the swell climate is validated on an offshore structural fatigue application

Keywords : Joint probabilities ; Metocean time-series ; Swell spectral models ; Ocean wave climate ; Event tracking ; Offshore fatigue design 


\section{Introduction}

In many applications, wave conditions need to be characterized by a history of directional spectra continuously provided for long durations of several years or decades. Those are applications where the period as well as the direction of incident waves matter, and where the interest bears not only on a few rare and extreme cases, but on frequent if not daily conditions. Typical examples are fatigue assessment of an offshore structure, prediction of profitability from the power output of a wave marine energy extraction device, study of coastal erosion of a shoreline, etc.

The present study is focused on offshore West Africa, mainly from Namibia to Nigeria. It makes use of the data and results of the WASP project (see Olagnon et al. (2004), summarized in Forristall et al. (2013) and companion papers, for thorough descriptions) and of some additional data measured off Angola also described in Guédé et al. (2010). Whereas at some locations, for instance the North Sea, most directional wave spectra are rather simple to model, with energy commonly concentrated around a single or at most two frequencies and directions, West Africa locations are submitted to much more complex wave conditions, with the presence at almost all times of several swells and of a wind sea, each of which pertaining to different frequencies and directions, thus multiplying the number of parameters that it is necessary to track. Direct determination of the joint occurrence probabilities of parameters that number to ten or so (individual heights, periods and directions of three to four simultaneous wave systems) from measured or hindcast data would require numerous observations of all possible combinations. Even with coarse discretizations for the parameters, millions to billions of sea states would need to be compiled to estimate those probabilities, i.e. thousands to millions of years, which is not commensurate with the time durations of measurements or hindcasts.

Further insight in the way wave systems are combined is necessary to estimate the joint probabilities of the various wave systems that prevail at a given time, and to model correctly their time evolution. As a consequence, conventional spectral shape models fail to represent those wave spectra. Moreover, when one wants to assess the long-term statistics of sea conditions, the observed data, that cover only short periods of time, do not allow to estimate the probabilities of occurrence of all the possible combinations of swells and wind sea (Guédé et al. (2010)). Hindcast wave data are not satisfactory either, since their calibration on far-propagated swells and wind seas raised by short squalls in inter-tropical areas is prone to substantial uncertainties. Hindcasts are also confined to reproduce the phenomena and models that their writers have chosen to put into them, and may misrepresent some natural features, see for instance chapter 9 of the WASP report Olagnon et al. (2004). 
The objective of the study is to define and assess parametric spectral models first for the individual sea state swell spectra, and then for the events, corresponding to remote storms, that gather consecutive observations of related wave systems over long durations, thus enabling estimation and use of longterm statistics of the swell climate. We intend in that manner to capture most of the dependencies between energy at different frequencies or directions in each individual spectrum, and between spectra at successive time-steps.

Only swell components are considered in the present study, yet wind seas could be studied in a similar manner. First, a method for the modeling of individual swells' directional spectra is described, and a triangular spectral shape is retained. Then, the modeling of the swell events in terms of their spectral parameters is presented. Lastly, a synthetic swell climate is reconstructed and validated on the lifetime assessment of an FPSO.

Some West Africa metocean data, measured with a directional waverider buoy anchored by $1450 \mathrm{~m}$ waterdepth off the coast of Angola, provides the practical basis for the study. Measurements at $1.28 \mathrm{~Hz}$ extend from March 2001 to March 2003, with some gaps due to sensor problems. Directional spectra were averaged over each 3 hour interval to ensure reasonable smoothing and partitioned using the SPOP software Kerbiriou et al. (2007).

\section{Modelling of individual swell directional spectra}

Spectra collected directly from wave observations at given locations consist in discrete spectro-directional densities (ie matrices sized by the number of frequencies and directional sectors). It is unpractical to use them in engineering applications because they represent a huge amount of data, show a large variability, and it cannot be verified whether any individual one is more appropriate than another one to characterize the long-term climate. The idea is thus to characterize the observed spectra by a small yet sufficient number of parameters from which the whole spectral shape can then be accurately reconstructed with an analytical curve.

\subsection{Conventional spectral models}

Many parametric spectral models have been proposed over the years for the one-dimensional (nondirectional) spectra. Some are simple and show one peak only. A general class of single peaked parametric models is the $\Gamma$-spectra:

$$
S(f)=A f^{-p} e^{-B f^{-q}}
$$


This form is popular for both wind waves and swell (Massel (1996)). Many models were derived from the $\Gamma$-spectra for different values of the exponents $p$ and $q$. A special subclass is obtained for $q=p-1$. Then, one gets the Pierson-Moskowitz spectrum for $p=5$, the Davidan spectrum for $p=5.5$, and the Davidan/Massel spectrum for $p=6$. Also, a $\Gamma$-spectrum where $q$ is set to 4 is called Wallops spectrum Huang et al. (1981).

The Pierson-Moskowitz Pierson $\&$ Moskowitz (1964) spectrum is one of the most widely accepted models, especially in medium or high latitudes. It corresponds to fully developed seas and has an infinite fourth spectral moment $m_{4}$, which raises some difficulties with narrow-bandwidth assumptions. For seas that have not reached the full development range, for instance because of a limited fetch, a better model is the one that was obtained by modifying it to represent the more narrow-banded sea-states of the North Sea in the Jonswap project after which it was named (Hasselmann et al. $(1973,1976)$ ). Though it was designed for the North Sea, the Jonswap model is used almost anywhere because the bandwidth can be adjusted by changing its peakedness parameter $\gamma$. However, there is little evidence to support its use when the climate is somewhat different from the North Sea one. Some authors also suggested the use of Gaussian or log-normal densities (Ewans et al. (2013); Forristall et al. (2013)).

To account for sea state spectra with many well separated peaks, several authors have suggested two-peaked models: Ochi $\&$ Hubble (1976) or Torsethaugen $(1993,1996)$ for instance. Those two-peaked models are usually built as the combination of two of the previous spectra, sometimes using some sensible constraints on the parameters in order to reduce their number. Yet, those models may be inadequate for a West Africa spectrum with several swell components (Figure 1).

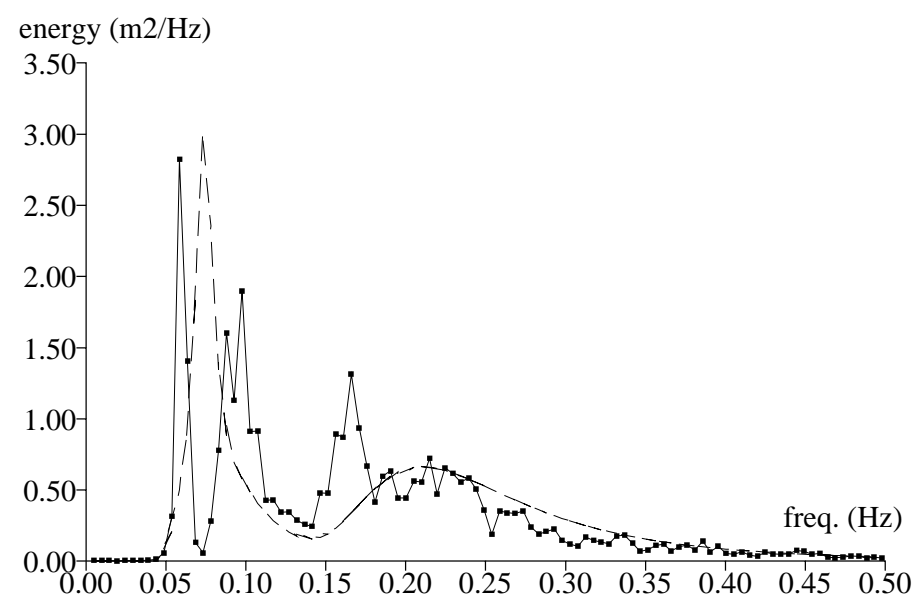

Fig. 1. A spectrum with 3 swell components (solid line) and its conventional model as the sum of two Jonswap (dotted line) 
All those models have the common feature that they were designed for wind seas in medium or high latitudes, and their extension to swell follows convenience arguments rather than physical analysis. Especially, much attention is given to the slope of the tail of wind sea spectra. Due to the celerity difference between long and short wavelengths and the finite fetch area and duration of the originating storm, a swell spectrum must on the contrary exhibit a cutoff frequency: too short waves relatively to the dominant wavelength cannot have arrived at the measuring point because they would have had to leave the storm area and thus have existed before the storm that created them (Gjevik et al. (1988)). Also several studies, see for instance the review in the report ISSC I.1 (2003), have shown the limitations of conventional spectral shapes when it comes to swell. Figure 1 shows how inadequate a conventional model may be for a West Africa spectrum with several swell components. On first examination, it appears clearly that a model for such a spectrum must be able to take into account three peaks or more.

A pragmatic consequence is that each peak of the spectrum should be modeled separately, allowing for a relatively large number of independent spectral peaks (for instance, three different swells and one wind sea), and that models designed for wind sea should only be used for wind seas. The impact of this partitioning of the spectra into several wave components can significantly improve the accuracy of the computed responses (see for instance Quiniou et al. (2003)).

\subsection{Proposed model for an individual swell spectrum}

Swell peaks are much narrower than the wind sea peaks that may be observed in the North Sea. Given that swells observed in the Gulf of Guinea were sent out by storms passing by in the Roaring Forties and never coming close to the location of interest, this can be verifed theoretically from propagation laws (Prevosto et al. (2013)). Modeling such narrow peaks by a Jonswap implies to have shape parameter $\gamma$ take much higher values than those in the commonly used range of 1 to 7 . For instance, it can be seen in chapter 11 of the WASP report (Olagnon et al. (2004)) that $\gamma$ is above 70 as soon as the swell period is above 8 seconds in the Northern Gulf of Guinea, and above 10 seconds off Angola or Namibia. Very high values of $\gamma$ for swell are somewhat in contradiction with the construction of the Jonswap shape, where that parameter is used to reflect the non-saturation of a fetch-limited wind sea whereas swell corresponds to the part of energy that propagated onto the location of observation, and they lead to a risk of unnoticed numerical inaccuracies in the practical computations. Moreover, the propagation of swells is not supposed to provide energy outside of a finite frequency interval. In order to keep the complexity of the fitting and reconstruction processes within reasonable limits, simpler 
formulations than the Jonswap one were thus also sought for, via the method described in Olagnon (2001) and recalled hereafter.

The assumption is made of a unique underlying spectral model, valid for all sea states, but undefined at this stage, of the form:

$$
S(f)=m_{0} F\left(\frac{f}{f_{c}}\right)
$$

where $f_{c}$ is a frequency characteristic of the sea state and $m_{0}$ the spectral moment of order 0 . Here, the characteristic frequency is set to the corresponding modal frequency, $f_{c}=f_{p} . F$ is normalized using $m_{0}$ so that:

$$
\int_{0}^{\infty} F(u) d u=1
$$

In the WASP report (Olagnon et al. (2004)), spectral bandwidth is modeled as $a f_{p}^{b}$, where $b$ takes values close to 2 in order to accomodate both swells and wind seas. However, if one limits oneself to swells, a mere linear dependency $a^{\prime} f_{p}$ is clearly sufficient. Coefficient $a^{\prime}$ varies with the latitude of the location, since it roughly represents the distance to the storm. When considering the spectral shape with respect to normalized frequency, the shape width appears thus constant for swells at a given location and it is reasonable to consider that so does the shape parameter of the spectral distribution.

In order to simplify the analysis and to give more chances of validity to the assumptions that the normalized shape would be the same for all sea states, only single-peaked spectral ranges need to be used, and swell conditions should be separated from wind sea conditions. For the swell frequency range in the spectra, this can be done rather easily by taking only the largest peak in the interval [12 20s] for analysis.

From the above unicity assumption and spectral estimation theory, it stems out that for each normalised frequency $\tilde{f}=\frac{f}{f_{p}}$, the observed spectral density is a random variable with a $\chi^{2}$ distribution with $\nu$ degrees of freedom of average value $F(\tilde{f})$.

$\nu$ is fixed, since we use the same smoothing/averaging scheme for each spectral estimate of the database, and as the $\chi^{2}$ distribution is fully defined by its parameter, all parameters of the distribution of the spectral densities (mean, mode, percentiles) should be in constant ratios with respect to each other and can be expressed as $C \times F(\tilde{f})$, if the above assumption that all have the same normalized shape is verified.

Figure 2 shows the resulting shapes for actual West Africa data. It was obtained with wave-staff measurements Welsh-averaged over records of 40 min- 


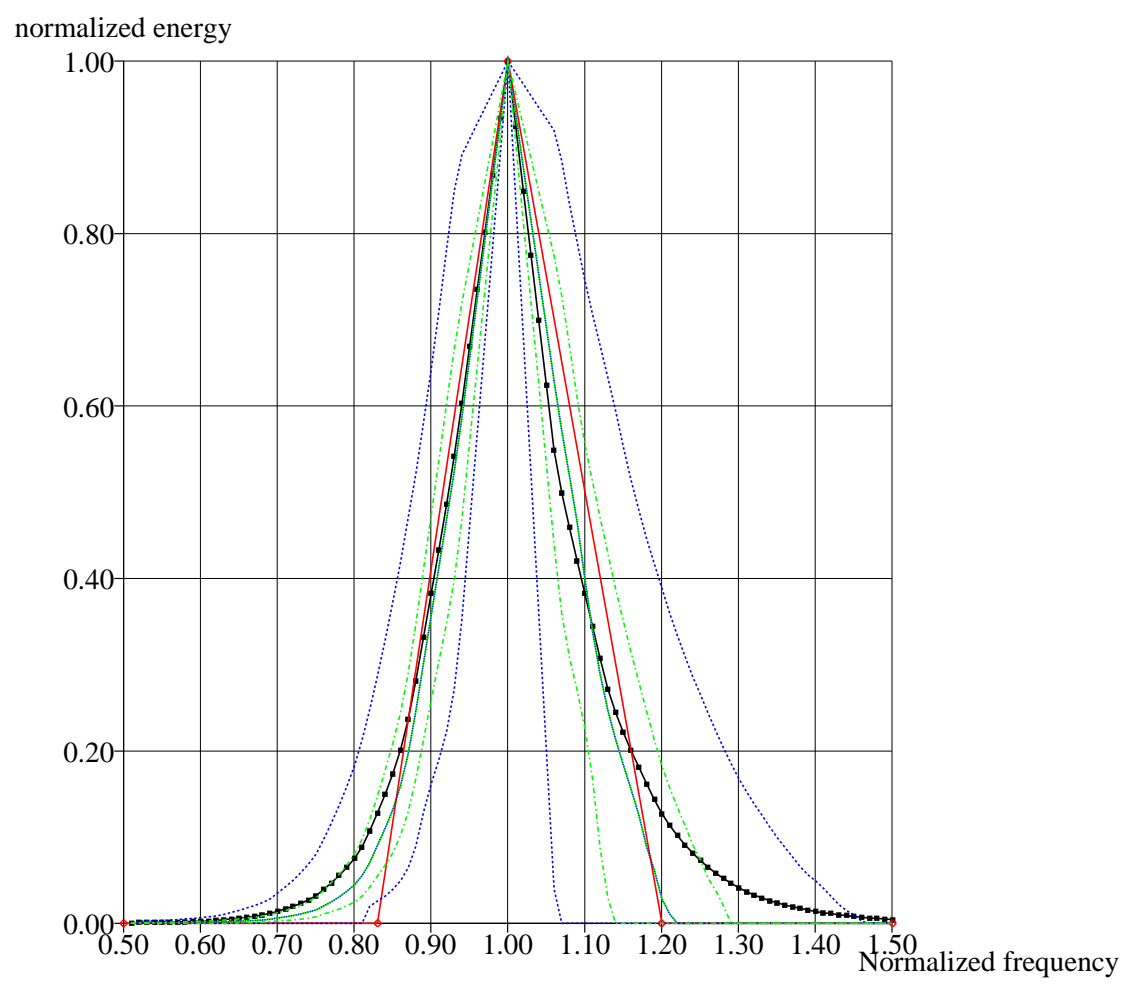

Fig. 2. Empirical shape, linear scale - green: median, 30\%, $70 \%$ percentiles, dotted blue: $10 \%$ and $90 \%$ percentiles, black: average, red: infered triangle

utes at the Ekoundou location (see Forristall et al. (2013)), thus more than 30 degrees of freedom each. Given that the result is not free from the influence of other neighbouring peaks, it was decided to use a triangular shape (displayed in red) to model the peak rather than some function that would follow more closely the black empirical one, and to assume that the deviation from that shape, especially at high frequencies, is mostly due to the influence of other wave systems and spectral estimation uncertainties. For the sake of simplicity, that triangle was chosen within the family of triangles extending from $\frac{\mu-1}{\mu} f_{p}$ to $\frac{\mu}{\mu-1} f_{p}$ and parameterized by the value $\mu$ :

$$
\begin{array}{ll}
T(f)=\frac{2 \mu(\mu-1)}{2 \mu-1} \frac{H_{s}^{2}}{16 f_{p}}\left(\mu \frac{f}{f_{p}}-(\mu-1)\right) & \frac{\mu-1}{\mu} f_{p}<f<f_{p} \\
T(f)=\frac{2 \mu(\mu-1)}{2 \mu-1} \frac{H_{s}^{2}}{16 f_{p}}\left(\mu-(\mu-1) \frac{f}{f_{p}}\right) & f_{p}<f<\frac{\mu}{\mu-1} f_{p} \\
T(f)=0 & \text { elsewhere }
\end{array}
$$

where $H_{s}$ stands for significant wave height and $f_{p}$ for peak frequency, see IAHR (1986) for usual notations regarding sea state parameters.

Note that $\mu$ can be estimated from the peakedness factor $Q_{p}$ as $\frac{3 Q_{p}+2}{4}$, with 


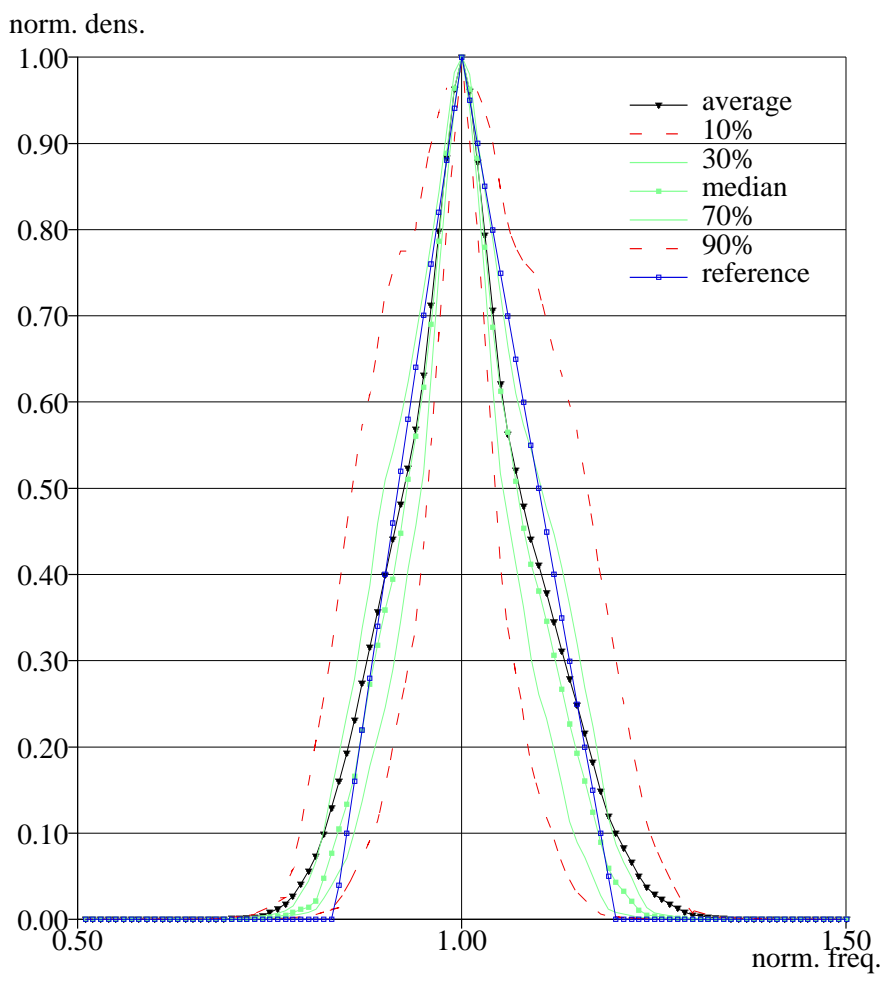

Fig. 3. Analysis of simulated spectra from triangular shape - green: median, $30 \%$, $70 \%$ percentiles, dotted red: $10 \%$ and $90 \%$ percentiles, black: average, solid blue: reference triangle

the usual definition of $Q_{p}$ :

$$
Q_{p}=\frac{2}{m_{0}^{2}} \int_{0}^{\infty} f S^{2}(f) d f
$$

Typical distributions of peakedness and directional spreading for West Africa locations can be found in the WASP report (Olagnon et al. (2004)). For swell systems identified in the database at the location of interest, $Q_{p}$ is about 12 and does not exhibit any statistically significant variation with frequency (correlation coefficient $\rho=0.03$ ), thus conforting the assumption that the shape parameter should be constant and that non-normalized bandwidth increases linearly with frequency.

Swell directional spreading at the peak frequency, with an angular standard deviation of 18 degrees ( $s \approx 16$ in the $\cos ^{2 s}$ form), exhibits only a variation of $0.2 \mathrm{deg} / \mathrm{s}$ with the wave period, and of little significance (correlation coefficient $\rho=0.08)$, thus it can also be taken as a constant for the database.

Now let us plot the averages and percentiles for synthetic data deriving from a single given spectrum of triangular shape assuming that it follows a $\chi^{2}$ 

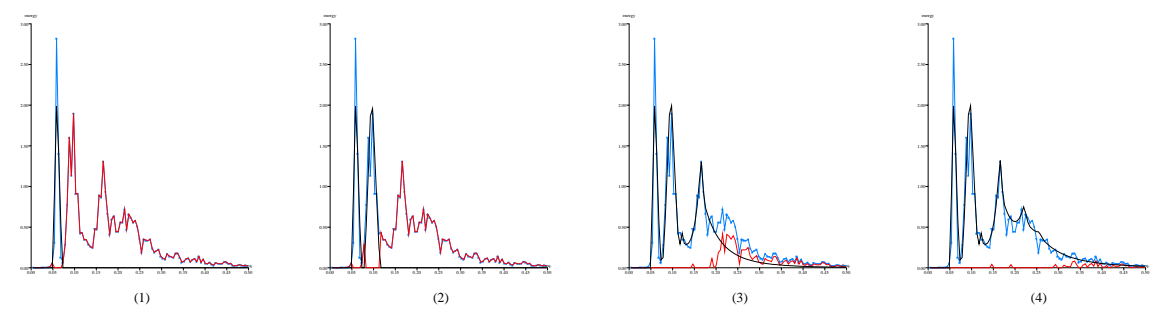

Fig. 4. The reconstruction process of a partitioned spectrum - blue: spectrum, black: reconstruction, red: residual

distribution (Figure 3). One can see that the obtained chart is similar to the one from the observed swell spectra (Fig. 2).

Figure 4 shows a partition of a typical West Africa sea state spectrum with a triangle model for swells and a jonswap model for the wind sea components. One can see how accurate that approximation is, compared to conventional models (Figure 1)).

\section{Modeling of the time-histories as swell events}

Our intent is to study the extension along the time dimension of the swell systems representations defined for individual spectra, so as to reproduce the underlying joint probability structure of the wave climate parameters. Such a procedure supplements conventional partitioning so as to enable to use the results to define joint occurrence probabilities and thus long-term climate specifications.

To this aim, we first define climate "events". A climate event is a phenomenon:

- that can be found in all successive observations within a finite, yet significant, time-duration;

- that can be modeled consistently, i.e. with the same model type, for each of those observations;

- for which the model parameters variations are slow and can be themselves modeled;

- and that can be traced back to a unique meteorological origin.

Once partitions are identified in each spectrum (see for instance Gerling (1991); Hanson 63 Phillips (2001); Ailliot et al. (2013)), one needs to associate them between consecutive time-steps when they represent the same event. Many authors use simple distance criteria in the parameter space $\left(H_{s}, f_{p}, \theta_{p}\right)$ where $\theta_{p}$ refers to the peak direction. Although ideally the criteria should follow physical considerations, for instance, observed $f_{p}$ of a swell system increases 
with time, see Prevosto et al. (2013), at this stage, coarse adjustment of the distance criteria is sufficient to isolate sets of systems that exist over many consecutive time-steps, and fromwhich we select the most representative ones that we will analyse in depth to characterize swell "events".

In the present study, systems were extracted and tracked using the SPOP software package described in Kerbiriou et al. (2007).

\subsection{Identification of a parametric model for a swell event's time history}

The problem of identifying parametric models for the time-evolution of the wave systems parameters is very similar to that of finding a parametric spectral shape for individual systems spectra. A similar procedure is thus used to solve it. Especially, swells that arrive to West African locations originate in the succession of very repetitive storms travelling eastwards in the Roaring Forties latitudes from Cape Horn or Argentina to Good Hope. Those storms are all of the same kind, every three days or so a new storm appears off Argentina and heads East, and this legitimates the assumption of a unique underlying model for those swell events (Prevosto et al. (2013)).

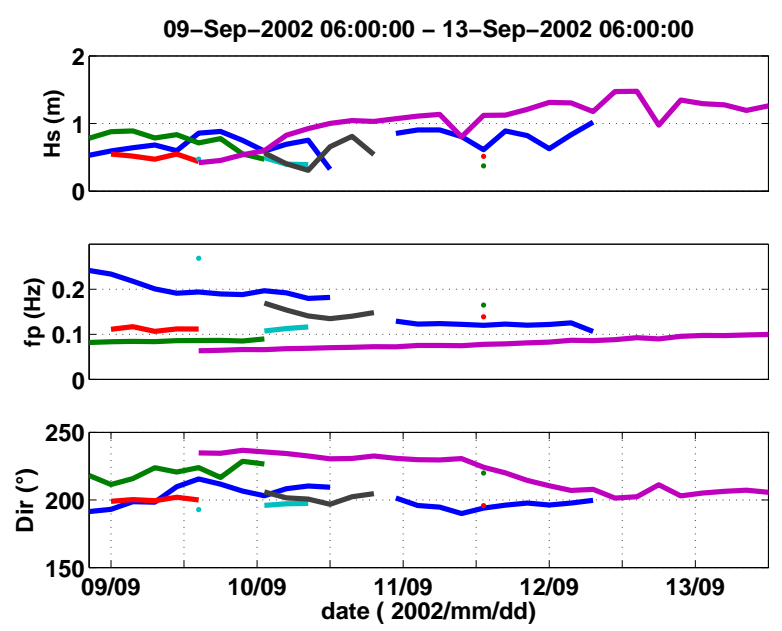

Fig. 5. Time-history of identified parameters

Given that we consider swell systems, the choice of the parameters to be monitored stems from the previous spectral model: $H_{s}, f_{p}$ and $\mu$ for the 1-D spectrum, $\theta_{p}$ and directional spreading for the directional characteristics. In practice, parametric models are needed only for the time-histories of $H_{s}, f_{p}$ and $\theta_{p}$, while $s$ and $\mu$ are taken as constants at the location of interest, as mentionned earlier. Figure 5 shows a 5 -day history of those parameters.

The first step is then to extract the swell events from those histories, i.e. to discard wind sea systems. One chooses first a threshold frequency, for instance 
$0.13 \mathrm{~Hz}$, and applies the criterion that any system above that frequency is not a swell system. As the climate in the area is exempt from strong local storms of any significant duration, since tropical squalls last only a few hours, wind seas are all eliminated with that criterion.

Although in a storm long waves are generated after the short ones, the wave celerity as derived from the dispersion equation implies that they arrive earlier to the West African locations and that the measured peak frequency increases with time (Prevosto et al. (2013)). Thus, in order to keep only representative systems for which the fitting of a model is meaningful, the systems are checked to exhibit a frequency increase consistent with the propagation celerity from an acceptable geographic location before they are marked as belonging to the same event. For instance, swells that would appear to originate from continental areas, or from areas known to have no storms of importance such as the Doldrums, are eliminated.

In a next step, events that are not suited to model estimation are discarded: those that did not last for more than 15 hours, and those where some obvious measurement problem has occurred, for instance with parameters remaining exactly constant over three consecutive observations at least. Out of the 618 events identified by the SPOP package in the database, only 165 were retained at this stage.

In order to make the remaining swell systems comparable, we set for each of them the time origin $t_{0}$ at the observation of maximum $H_{s}$. This also has the advantage that the estimation of the characteristics is then at its best, as the quality decreases when the energy decreases.
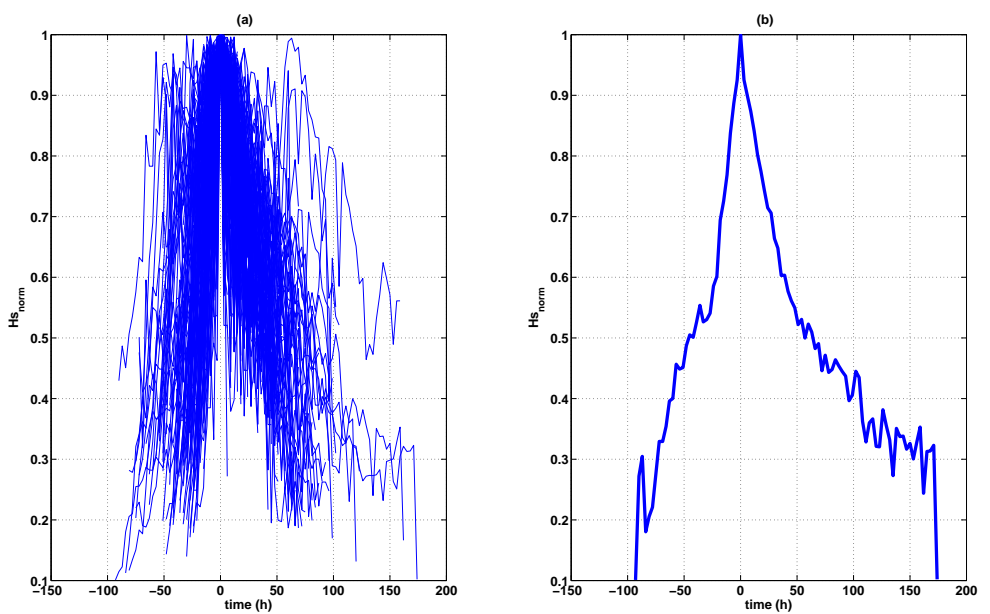

Fig. 6. Plot of normalized events: (a) all retained events, (b) median

A model may then be sought for the time history of an event's $H_{s}$ in the same manner as for spectral shapes. Normalization is carried out by the maximum 
$H_{s}$ of the event to bring all histories to 1 at their time-origins, as shown on figure 6 (a). No normalization is applied to the elapsed time scale. Examination of figures 6 (a) and (b) suggests the choice for $H_{s}$ of linear ascending and descending models, and to give little importance to the deviations from these at the very start and end of the event since they usually correspond to poorly estimated values. The slopes of the ascending and descending parts are thus fitted using weighted least squares with weights $\frac{1}{\left|t_{i}-t_{0}\right|}$, where the $t_{i}$ are the times of the observations.

Frequency can be expected to increase linearly along an event, and its slope is determined using again weighted least squares fit, with weights set to the corresponding $H_{s}$ values.

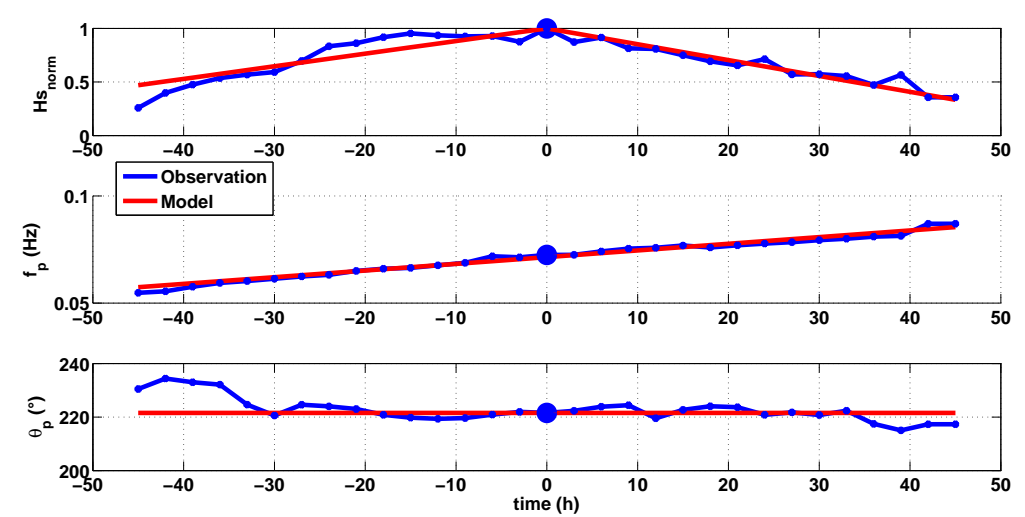

Fig. 7. An event and its model

Direction turns slowly anticlockwise as the originating storm moves from Cape Horn to Good Hope. Yet, for the sake of simplicity, since the drift in direction over the event's duration is small with respect to the direction discretization steps commonly used in engineering applications, direction was assumed constant over an event in the present study. Figure 7 shows an example of a modeled event in terms of $H_{s}$, frequency and direction. This requires 6 parameters: maximum $H_{s}$ values, $H_{s}$ growth slope, $H_{s}$ decay slope, $f_{p}$ value at time of maximum $H_{s}, f_{p}$ slope, direction.

The event duration is determined by the ascending and descending $H_{s}$ slopes, and thus an individual event is fully determined from the above 6 parameters.

\subsection{Joint distributions of swell events model parameters}

Joint distributions were estimated for pairs of parameters and their correlations computed. Figure 8 shows those for which correlations are statiscally significant. 

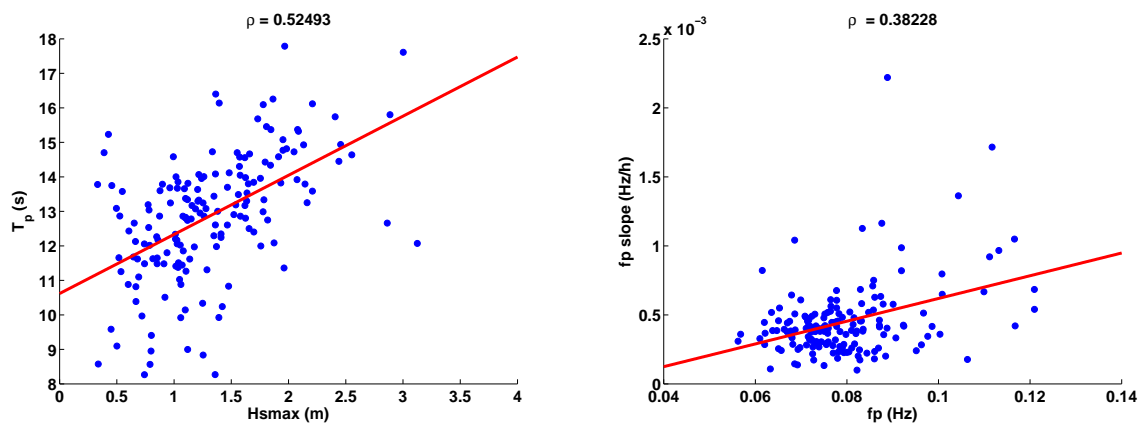

Fig. 8. Joint distributions of event maximum $H_{s}$ and period at that instant (left), of corresponding frequency and frequency growth slope (right)

Apart from the maximum $H_{s}, f_{p}$ (here displayed with $T_{p}=\frac{1}{f_{p}}$ to obtain a more linear relationship) and the $f_{p}, f_{p}$ slope diagrams, correlations are not statistically significant. The structure of the joint probabilities can thus be represented by the marginal distribution of maximum $H_{s}$ in the event, the distribution of $f_{p}$ at the time of maximum $H_{s}$ conditional on that value of $H_{s}$, the distribution of $f_{p}$ growth slope conditional to the value of $f_{p}$ at the time of maximum $H_{s}$, and marginal distributions for the other parameters that were found independent.
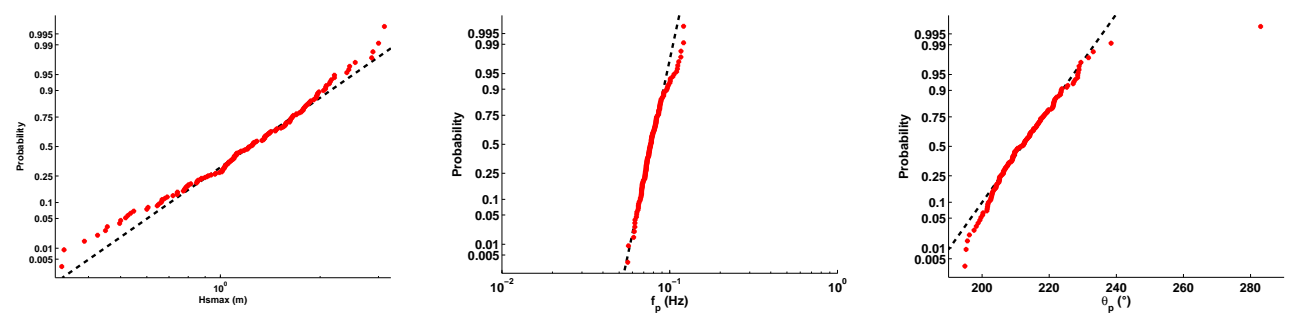

Fig. 9. Marginal distributions of maximum $H_{s}$ (left), frequency at maximum $H_{s}$ (center) and direction (right)

The main parameters' marginal distributions are shown on figure 9. Attempts were made to fit them with several parametric models, namely Gumbel, Gaussian, log-normal. In most cases the best fit was obtained for a log-normal model. For the descending $H_{s}$ slope, the behaviour looked different for small and high values, as if two populations were mixed, and the best fit was obtained by the sum of two log-normals. Yet, it should be verified with other data when available that the population corresponding to steepest descents is not an artefact due to the lost of systems in the tracking because of gaps in the measurements or merging into local wind seas. The distributions are as follows:

- Maximum $H_{s}$ : log-normal distribution.

- Ascending $H_{s}$ slope: log-normal distribution.

- Descending $H_{s}$ slope: sum of 2 log-normal distributions.

- $f_{p}$ : log-normal distribution. 
- $f_{p}$ slope: log-normal distribution.

- $\theta_{p}: 99 \%$ truncated normal distribution, with discrete addition. Most swell systems come from the Southwest sector (South Atlantic), yet on rare but verified instances (about 1\%), Northern Hemisphere swells reach the location where they arrive from the Northwest.

\section{Construction of synthetic series of swell spectra}

\subsection{Procedure}

In order to construct the swell climate, we need to supplement the model for individual swell events with a model for their occurrences. A straightforward idea is to assume that events happen independently of each other and to estimate, from the database, the distribution of the time-durations between the $H_{s}$ maxima of successive events. A good fit can be obtained with the sum of two log-normal distributions, one modelling events separations below 36 hours, the other separations longer than 36 hours. Yet reconstructed climate with that method is not acceptable: many identified events separated by less than 36 hours were partial fragments of longer events, and when they are simulated as full events, their average number at each time step and the total energy time-density are too high in the simulation. Durations between the events that were found of sufficient quality are not representative of the actual durations if all events could be correctly identified (via a manual process). Even calling on hindcast data, less prone to but not exempt of tracking failures in the partitioning process, does not satisfactorily solve the problem.

Further study of the process of events and of their inter-dependence is thus required. This is the subject of on-going work, and the coarse method presented here aims only at demonstrating the feasability of reconstruction and at assessing the event model developed above. It should be a sufficient approximation for applications only weakly sensitive to the order of the sea state sequence, but would fail to reproduce seasonality and rare occurrences, and would be disputable for the estimation of the joint conditions leading to response extremes for instance. Yet for fatigue studies that consider the main bulk of the sea states distribution and where order has little influence, although the process of the events is ill-known, some realistic simulated histories may still be obtained in the following manner:

- Assume a given distribution shape for the durations between successive events. Since measured durations could be approximated with log-normals, we used also a sum of two log-normal distributions.

- Adjust the parameters of that distribution so as to meet the two conditions 
a) the average number of events present at each time-step should match the average number of systems that the partitionning software identifies on the measured data off Angola, 1.56, and b) the overall root mean square of $H_{s}$ should be the same as the measured value, i.e. $1.3 \mathrm{~m}$. Those values are computed over the whole database and are thus not affected by the event selection process. Energy density or some other quantity could be prefered to mere $H_{s}$ for some applications, yet a simple study of energy flux

$\frac{H_{s}^{2}}{f_{p}}$ over 30 years of hindcast at location $10 \mathrm{~S}-10 \mathrm{E}$ shows that $H_{s}$ has less interannual variability ( $8 \%$ versus $24 \%$ ), and is thus a parameter less prone to estimation errors from short measurement campaigns. In practice, we reduced the proportion of short intervals by changing the relative weights of the two log-normals.

- Simulate independent occurrences of events with those intervals.

\subsection{Validation}

In order to further assess the reconstruction method, tests were carried out with the industrial application of Guédé et al. (2010). Fatigue damage due to the vertical bending moment of an FPSO was computed, using the actual directional transfer functions that had been calculated for its design and an arbitrarily scaled stress concentration factor, so as to use the two-slope fatigue $\mathrm{S}-\mathrm{N}$ curve in a realistic manner. On the one hand, the swell part of the observed directional spectra over the validated 1.64 year of measurements were used as input. On the other hand, 60 simulations were carried out with the above parameters for 1.64 years of time-history, triangle spectra were reconstructed and added from the systems time-history, and the fatigue damages calculated with this input.

Since the time-steps in the database with no identified swell systems were assumed to correspond to measurement failures, whereas some occur in the simulation, there was some doubt as to the root mean square $H_{s}$ value to be taken as the target. It was decided to aim in the simulation at the observed value of $1.28 \mathrm{~m}$ as conditional average on the presence of at least one swell system. Similarly, the average number of systems present at each time-step is not robust with respect to detection of starting and ending events in presence of other ones, so that condition was only checked for sensible results and not exactly. A satisfactory result was obtained for durations between events following the weighted $(0.14,0.86)$ sum of two log-normals with medians at 3.5 and 4.5 days. Those medians and weights were determined from a large number of attempts through a trial and error process and are stable with respect to the random seed of the simulation.

Table 1 summarizes the comparison between applications of the reconstructed 
Table 1

\begin{tabular}{|l|c|c|}
\hline Property & Observed & Reconstructed \\
\hline$H_{S R M S}$ & - & $1.21 \mathrm{~m}$ \\
$H_{S R M S} \mid N_{\text {swell }} \geq 1$ & $1.28 \mathrm{~m}$ & $1.28 \mathrm{~m}$ \\
$H_{S R M S} \mid N_{\text {swell }} \geq 2$ & $1.29 \mathrm{~m}$ & $1.48 \mathrm{~m}$ \\
interannual c.o.v. $\left(H_{S R M S}\right)$ & $8 \%$ & $9 \%$ \\
$\overline{N_{\text {swell }}}$ & 1.56 & 1.32 \\
$\overline{T_{p}} \mid N_{\text {swell }} \geq 1$ & $12.1 \mathrm{~s}$ & $12.2 \mathrm{~s}$ \\
$\overline{\left(\frac{H_{s}^{2}}{f_{p}}\right)} \mid N_{\text {swell }} \geq 1$ & $20.8 m^{2} / \mathrm{s}$ & $21.1 \mathrm{~m}^{2} / \mathrm{s}$ \\
1.64 year swell damage & $1.410^{-4}$ & $1.310^{-4}$ \\
c.o.v. damage & - & $75 \%$ \\
\hline
\end{tabular}

Comparison of the statistics of observed and reconstructed 1.64 year series of sea states

and observed swell histories to the FPSO fatigue case. For the simulated series, the median fatigue damage over the 60 simulations is given. It is clear that the statistical variability of damage, the reconstructed history is satisfactory for that application.

Although only the $H_{S}$ density was taken as a target objective when choosing the distribution of durations between events, the average values of peak periods and of energy density are well reproduced, providing an indirect validation of the individual event model that links them to $H_{S}$.

\section{Discussion and conclusions}

We have developed a model for swell events at West Africa locations. This model is consistent with the correspondence between swell events and remote storms. It provides parametric shapes for the joint time-histories of significant wave height, dominant wave period and main wave direction at the location of interest. Significant wave height is modeled by a maximum value, a linear growth and a linear decay of independent slopes. Dominant period is modeled by its value at the time of maximum $H_{S}$ and by a single slope for linear increase. Main direction is assumed constant given the accuracy usually required for engineering applications, although a slight counterclockwise rotation could also be considered.

Statistical distributions of the relevant parameters and their correlations are also provided. Reliable estimation of those parameters distributions will require to merge the contributions from the field data and from hindcast, and 
to integrate the event model into the partitioning and tracking software, and was not yet attempted within the scope of this paper.

Using a simplistic scheme for the occurrence process of the swell events, long realistic time-histories could be reconstructed that reproduce satisfactorily the swell climate for a structural fatigue calculation for an offshore structure.

Further research should enable to improve the modeling of that occurrence process and to extend the method to wind sea wave systems. Other time series, such as for instance current, temperature, salinity profiles in the case of oceanography, could benefit from a similar approach.

Now that a model for the time-history of those events has been defined, the tracking phase might also be improved by looking for their presence rather than only pairing systems in consecutive observations.

\section{References}

Ailliot P., Maisondieu C., Monbet V. (2013) Dynamical partitioning of directional ocean wave spectra. Probabilistic Engineering Mechanics. Vol. 33, pp. 95-102.

Ewans, K., Forristall, G.Z., Olagnon, M. \& Prevosto, M. (2013) Response sensitivity to swell spectra off West Africa, Proc. 32nd Int. Ocean, Offshore and Arctic Eng. Conf., OMAE 2013-11252

Forristall, G.Z., Ewans, K., Olagnon, M. \& Prevosto, M. (2013) The West Africa Swell Project (WASP), Proc. 32nd Int. Ocean, Offshore and Arctic Eng. Conf., OMAE 2013-11264

Gerling, T.W. (1991) Partitioning Sequences and Arrays of directional Ocean Wave Spectra into Component Wave Systems, J. Atmospheric and Oceanic Technology, Vol. 9, pp 444-458.

Gjevik, B., Krogstad, H.E., Lygre, A., Rygg, O. (1988) Long Period Swell Events on the Norwegian Shelf, J. Phys. Ocean., 18, pp. 724-737.

Guédé, Z., Olagnon, M., Pineau, H., François, M. \& Quiniou, V. (2010) Fast spectral rainflow fatigue damage assessment under wideband multipeak loading, Proc. Prads Conf., Rio de Janeiro.

Hanson, J.L., Phillips, O.M., Automated Analysis of Ocean Surface Directional Wave Spectra (2001) Journal of atmospheric and Oceanic Technology, Vol. 18, pp 277-293.

Hasselmann, K, et al. (1973) Measurements of wind-wave growth and swell decay during the Joint North Sea Wave Project, Deutsche Hydrographische Zeitschrift, Reihe A 8 (12), 95 pp.

Hasselmann, K., Ross, D.B., Müller, P. \& Sell, W. (1976) A parametric wave prediction model, J. Phys. Oceanogr., Vol. 6, no. 2, pp. 200-228.

Huang, N.E.. Long, S.R., Tung, C.-C., Yuen, Y. \& Bliven, L. (1981) A uni- 
fied two-parameter wave spectral model for a general sea state, J. Fluid Mechanics, Vol. 112, pp. 203-224.

International Association for Hydraulic Research (1986) List of Sea State Parameters Suppl. to Bulletin No 52

ISSC, A. Mansour \& R. Ertekin eds. (2003) Report of Technical Committee I.1 - Environment Proc. 15th ISSC, Vol. 1.

Kerbiriou, M.-A., Prevosto, M., Maisondieu, C., Babarit, A. \& Clément, A. (2007) Influence of an Improved Sea State Description on a Wave Energy Converter Production, Proc. 26th Int. Ocean, Offshore and Arctic Eng. Conf., OMAE 2007-29254

Massel, S.R. (1996) Ocean Surface Waves, their Physics and Prediction, World Scientific.

Hubble, E.N., Ochi, M.K. (1976) On six-parameter wave spectra, Proc. 15th Coastal Engineering Conference, American Society of Civil Engineers (ASCE), pp. 321-328.

Olagnon, M. (2001) Representativity of some standard spectral models for waves, Proc. Int. Offshore and Polar Engineering Conf., ISOPE Vol. 3, pp. 92-99.

Olagnon, M., Prevosto, M., Van Iseghem, S., Ewans, K. \& Forristall, G.Z. (2004) WASP - West Africa Swell Project - Final report and Appendices, See URL http://archimer.ifremer.fr/doc/00114/22537/

Olagnon, M., Ewans, K., Forristall, G.Z. \& Prevosto, M. (2013) West Africa Swell Spectral Shapes, Proc. 32nd Int. Ocean, Offshore and Arctic Eng. Conf., OMAE 2013-11228

Pierson, W.J. \& Moskowitz, L. (1964) A proposed spectral form for fully developed wind seas based on the similarity of S.A. Kitaigorodskii, J. Geophys. Res., Vol. 69, no. 24, pp. 5181-5190.

Prevosto, M., Ewans, K., Forristall, G.Z. \& Olagnon, M. (2013) Swell genesis, modelling and measurements in West Africa, Proc. 32nd Int. Ocean, Offshore and Arctic Eng. Conf., OMAE 2013-11201.

Quiniou-Ramus, V., Hoche, M.-A., François, M., Nerzic, R., Ledoux, A., Orsero, M. (2003) Recent Breakthroughs in the Analysis of Total E\&P Angola Block 17 wind/wave/current records and their impact on floating structures design, Proc. XVth Deep Offshore Technology Conf., DOT, Marseilles.

Torsethaugen, K., A two Peak wave spectrum model (1993) Proc. Int. OMAE Conf., Vol.2, pp. $175-180$.

Torsethaugen, K., Model for a double peak wave spectrum (1996) Report SINTEF SFT22 A96204, ISBN 82-595-9223-1, 13 pp. 